\title{
Linguistic features of the therapeutic alliance in the first session: a psychotherapy process study
}

\author{
Attà Negri, ${ }^{1}$ Christopher Christian, ${ }^{2}$ Rachele Mariani, ${ }^{3}$ Luca Belotti, ${ }^{1}$ Giovanbattista Andreoli, ${ }^{1}$ Kerri Danskin ${ }^{4}$ \\ ${ }^{1}$ Department of Human and Social Sciences, University of Bergamo, Bergamo, Italy; ${ }^{2}$ City College of New York, New York, NY, USA; \\ ${ }^{3}$ Italian Psychoanalytic Society, Rome, Italy; ${ }^{4}$ Princeton University, Princeton, NJ, USA
}

\begin{abstract}
Critical aspects of the therapeutic alliance appear to be established as early as the first session. Specifically, the affective bond between the therapeutic dyad appears to develop early in treatment and tends to remain stable over time, while agreements on goals and tasks tend to fluctuate over the course of treatment. Are there distinguishable early signs of a strong therapeutic alliance? In this study, we examined how some linguistic measures indicative of joint emotional elaboration correlated with a measure of the therapeutic alliance assessed within a single session. Initial intake sessions with 40 patients with varying diagnoses were videotaped, transcribed, and analyzed using linguistic measures of referential process and then scored with the Segmented Working Alliance Inventory-Observer form. Results showed that patients who were rated as more emotionally engaged in relating their experiences and then reflecting on them by mid-session also had higher scores in the therapeutic alliance by the final part of that same session. An implication of this study is that the interpersonal factors facilitating elaboration of inner experience, including elements of warmth, safety, and analytic trust, are related to the development of early therapeutic alliance. These findings did not appear to be dependent on the patient's psychopathology. This study is one in a growing line of research exploring how patients speak rather than just the content of what they say.
\end{abstract}

Key words: Therapeutic alliance; Referential process; Referential activity; Therapeutic linguistic dimensions.

Correspondence: Attà Negri, Department of Human and Social Sciences, University of Bergamo, Piazzale S. Agostino 2, 24129, Bergamo, Italy.

Tel.: +39.035.2052914.

E-mail: atta.negri@unibg.it

Citation: Negri, A., Christian, C., Mariani, R., Belotti, L., Andreoli, G., \& Danskin, K. (2019). Linguistic features of the therapeutic alliance in the first session: a psychotherapy process study. Research in Psychotherapy: Psychopathology, Process and Outcome, 22(1), 71-82. doi: 10.4081/ripppo.2019.374

Contributions: the authors contributed equally.

Conflict of interest: the authors declare no potential conflict of interest.

Funding: none.

Dedication: dedicated to Jeremy Safran.

Received for publication: 21 January 2019.

Revision received: 3 March 2019.

Accepted for publication: 4 March 2019.

This work is licensed under a Creative Commons Attribution NonCommercial 4.0 License (CC BY-NC 4.0).

CCopyright A. Negri et al., 2019

Licensee PAGEPress, Italy

Research in Psychotherapy:

Psychopathology, Process and Outcome 2019; 22:71-82

doi:10.4081/ripppo.2019.374

\section{Introduction}

Over the last two decades, an increasing number of empirical studies have highlighted the clinical and heuristic usefulness of the therapeutic alliance construct. Research on the alliance has underscored how interpersonal factors can contribute to psychotherapeutic success more than technical and theoretical factors (Lambert, 2013; Lambert \& Barley, 2001). And by now it is well established that the therapeutic alliance is the best predictor of therapeutic outcome (Castonguay, Constantino, \& Holtforth, 2006). Flückiger, Del Re, Wampold, and Horvath (2018), analyzing 295 independent studies involving more than 30,000 patients between 1978 and 2017, found a robust positive relationship between alliance and treatment outcome, which remains consistent across assessors' theoretical perspectives, alliance and outcome measures, treatment approaches, patient characteristics, and nationalities. Several other studies have focused on how alliance ruptures can be usefully addressed and used to better understand the patient's functioning, help foster the therapeutic relationship, and improve treatment outcome (Colli, Gentile, Contino, \& Lingiardi, 2017; Safran, Muran, \& Eubanks-Carter, 2011).

While the therapeutic alliance is undoubtedly recognized as a central element of the psychotherapeutic process and treatment outcome, there is still much to be learned about the processes leading to the development of that alliance, particularly as it relates to technical skills and techniques that clinicians can use to foster the alliance and help repair ruptures. 
In this regard, the findings obtained by Eubanks, Muran, and Safran (2018) that identified specific researchsupported practices for addressing and repairing ruptures in the alliance are noteworthy. These include acknowledging the rupture directly; inviting patients to explore their experiences of the rupture; empathizing with patients' negative feelings about the therapist or the therapy; and anticipating that ruptures can evoke feelings of confusion, incompetence, and guilt in some therapists. These results highlight an implicit aspect of the alliance and, more generally, of the therapeutic relationship - mainly, the importance of focusing on the interpersonal, emotional, and affective process of therapy. In spite of that, the enhancement and preservation of the therapeutic alliance, the helping alliance, or the working alliance are usually intended as an explicit aim of therapy - a tool that the therapist deliberately employs, mainly through specific attention to the presence of markers of alliance ruptures and purposeful attempts at resolutions. In a study by Johnsson and Stenlund (2010), the authors distinguish between what they call rational elements of the alliance (e.g., agreement on task and goals) and the affective dimension of the alliance (e.g., the quality of the emotional bond). The authors argue that a focus on the affective dimension of the alliance, more so than on the rational dimension, is what accounts for change in treatment. Also Safran and Kraus (2014) described some metacognitive processes that could enhance alliance development, but they concluded that the techniques of metacommunication are not to be confused with the essence of the skill of resolving alliance ruptures; rather, in order to foster the alliance the most important skill for therapists to hone is "to internally track what is emerging in the therapeutic relationship on a moment-by-moment basis, to be able to reflect on what may be playing out in the treatment" (p. 385).

Safran and Muran's (2000) definition of alliance, consistent with contemporary psychoanalytic relational thinking (e.g., Aron, 1996; Mitchell, 1998; Safran, 2012), construes the alliance as a continuous negotiation within the therapeutic dyad at both conscious and unconscious levels. The alliance represents, therefore, an emergent property of the relationship, instead of an aim or a technique. It is an ongoing interpersonal challenge that continues throughout the entire process of treatment and operates on both explicit/rational and implicit/affective levels. Thus the importance of monitoring what is being enacted relationally between therapist and patient on an ongoing basis in order to understand the development of the alliance and its natural fluctuations.

\section{Referential process}

One of the more promising ways to study the interactive processes that characterize the evolution of the therapeutic relationship is to analyze the words used by the patient and the therapist. Both the verbal contents and, in particular, the linguistic style reveal significant informa- tion about what is occurring interpersonally between the dyad in terms of emotional engagement and reflection both core mechanisms of change. From Bucci's Multiple Code Theory (MCT; Bucci, 1997) we draw a useful conceptual framework and some tools to analyze the relationship between the therapeutic alliance and the mental processes of participants in the clinical exchange, as mapped by linguistic markers.

The MCT is a general theory of emotional information processing that is derived from current work in cognitive psychology, psychoanalysis and affective neuroscience (Bucci \& Maskit, 2007). According to Bucci (1997; 2000), human beings use three basic ways to elaborate information and build up images and representations: i) the subsymbolic; ii) nonverbal symbolic; and iii) verbal symbolic processing systems. In the subsymbolic system multiple information is processed simultaneously in a global and analogical modality, along continuous dimensions, which often constitutes the affective core according to Emde (1983). This system is involved in recognizing nonverbal communication - a crucial process in the patienttherapist relationship - as well as in many other common actions such as identifying a familiar voice, doing physical exercises, or performing creative work. We propose that these nonverbal aspects are strictly connected to the intersubjective process involved in building the therapeutic alliance, beginning as early as the intake session. The nonverbal symbolic system operates with discrete images or representations that take shape from the continuous flow of subsymbolic experience. Finally, in the verbal symbolic system these images and representations can be translated into words, even if only partially; this system allows for abstract thought, reflection, and communication with others.

Bucci (1997) proposes that these three types of processing systems are interconnected by means of what she calls referential process (RP), which functions to transform subsymbolic experiences into nonverbal symbolic form, and then into language. Bucci theorizes that in doing so, RP makes it possible to communicate one's emotional experience to other people, to understand others through their own words, and to self-regulate or mutually regulate with a partner or group through one's own or other people's words. Mental images facilitate the connection of multimodal, subsymbolic experiences into articulated thoughts. Bucci posits that RP in the context of psychotherapy operates to change emotional schemas through sequential occurrence and reiteration of three major phases: i) arousal; ii) symbolizing in narrative, and iii) reorganizing (Bucci, 1997; 2002a; 2002b; 2005; Bucci, Maskit, \& Murphy, 2016; Mariani, Maskit, Bucci, $\&$ De Coro, 2013). These phases are described as follows:

i) Arousal. A problematic emotion schema is activated; the patient appears to be struggling with painful feelings and ideas associated with the schema. The subsymbolic processing system, involving bodily and 
sensory experience, is dominant; this constitutes the affective core of the schema.

ii) Narrative/Symbolizing. A specific experience emerges that represents aspects of the problematic schema that the patient can express verbally in the form of a narrative of an event, or a memory, dream or fantasy. This represents the essential symbolizing process, connecting the sub-symbolic flow of experience to language. The speaker must be emotionally engaged in the schema to enable the symbolizing process to operate effectively in expressing emotion verbally. The emotion schema may often play out in the relationship as well.

iii) Reorganizing. Once the schema has been experienced and represented as a narrative, or played out in the relationship, therapeutic interventions can then come into play to interpret, explore and reflect upon the issues that are being expressed. Herein lies the potential for new connections and changes in emotional organization to be made.

Effective sessions are likely to be characterized by relatively well-organized appearances and reiteration of the RP phases; in less effective work, the process does not play out, or plays out incompletely (Bucci, 1997; 2002a; 2002b; 2005; Bucci, Maskit, \& Murphy, 2016; Kingsley, 2010; Kris, 1956).

Over the last 20 years, several linguistic measures have been developed and validated in different languages to visualize, monitor, and measure these phases of the referential process. Initially they consisted of hand coding of the therapeutic conversation along specific dimensions (Bucci, Kabasakalian, \& the Referential Activity Research Group, 2004); subsequently, a computerized program has been developed to allow for automated coding (Maskit, 2011; Maskit, Bucci, \& Murphy, 2012) by comparing speech with specific linguistic dictionaries.

The two more important measures of the referential process are the Referential Activity (RA) and the Reflection and Reorganization function (RR):

i) RA can be defined as the degree to which the speaker or writer is able to translate their emotional, visceral and relational experience into words, so as to evoke corresponding experiences in the listener or reader (Bucci, 1984; Bucci \& Freedman, 1978; Bucci, Kabasakalian, \& the Referential Activity Research Group, 2004). The more concrete, specific, clear, and vivid speech is, the more the words work to translate the subsymbolic experience in an immediate way for the interlocutors. This measure is crucial to detect and monitor the narrative/symbolizing phase of RP.

ii) RR can be defined as the degree to which the speaker is trying to recognize and understand the emotional significance of an event or set of events in their own or someone else's life, or in a dream or fantasy (Bucci, Zhou, \& the Referential Activity Research Group, 2018; Negri et al., 2018). It is not an abstract and logical reasoning but an active searching for subjective meanings of a memory in which the speaker is emotionally engaged. This measure allows for visualization and monitoring of the reorganizing phase of referential process.

Other complementary measures of the referential process have also been developed. They detect the proportion of words present in a speech sample related to specific themes connected in some way to the RP such as affects, abstract reflection, sensory-somatic issues, and disfluent items. They are described below in the measures section. Details of their development are presented elsewhere (Bucci \& Maskit, 2006, 2007; Mariani et al., 2013; Negri et al., 2018).

\section{Therapeutic alliance and referential process in the intake session}

In this study, we integrate these two different lines of process research (i.e., alliance research and referential process research) in order to better understand the initial construction of the therapeutic alliance in the first intake session and how subsymbolic, emotional, and reflecting experiences might contribute to this process. Critical aspects of the therapeutic alliance appear to be established as early as the first session (Horvath, Del Re, Flückiger, \& Symonds, 2011; Sexton, Littauer, Sexton \& Tømmerås, 2005). Specifically, the affective bond between the therapeutic dyad appears to develop early in treatment and tends to remain stable over time, while agreements on goals and tasks tend to fluctuate over the course of treatment (Horvath, 2005; Zilcha-Mano \& Errázuriz, 2017). The purpose of our study is to discover whether there are any preliminary signals under the surface of formal linguistic interaction taking place in those early interactions of the first intake session. As described above, Safran and Muran (2000) proposed that the psychodynamic relational matrix plays an important role in building a good working alliance. In an emotional and verbal exchange, the therapeutic dyad continuously negotiates, on conscious as well as unconscious levels, co-creating the foundation of a working relationship from which meaningful change can emerge. The alliance represents, therefore, an emergent property of the relationship, not simply an aim or a technique - one that theoretically should be observable in the variations in emotion and reflection in patient-therapist dialogue. In other words, the working alliance can be viewed as a variation of linguistic style. So, in this study, we explore how linguistic style - the manner in which patient and therapist talk to each other - measured by sophisticated computerized linguistic measures in the frame of referential process can reveal different characteristics of moments of rupture and repair.

We know that intake sessions differ from the subsequent treatment sessions in many aspects, such as the greater uncertainty in interpersonal positioning and expectations of both participants, the patient's state of suffering, the greater concentration on the description of 
symptoms and problems. Moreover, the patient tends to be very emotionally activated because they are entering a setting that intensifies expectations with respect to a desired change. For all these reasons, we expect that the measures of RA will remain moderately high, even when the patients are expected to be focusing on the description of their symptoms. In one study (Okie, 1992) that explored the relationship between somatization, acting out, and verbalization in a sample of 50 female inpatients with Borderline Personality Disorder, the author found a positive correlation between these content areas and referential activity. These findings challenged the notion that somatization and symptom talk is performed in the service of defense and leads to less emotional engagement.

We hypothesize a positive correlation between a patient's capacity to be in contact with emotional experience, being immersed in the storytelling and reflecting on it, and the quality and strength of the therapeutic alliance. That is, we predict a positive correlation between alliance and referential process. Interpersonal factors, like warmth, safety, and analytic trust, elements that well describe the therapeutic alliance, can facilitate the patient's ability to explore and make contact with inner experience. However, we assume that the linguistic measures denoting reflection and reorganization upon emotional activation are what account for the difference between high and low alliance scores - and not the RA scores alone, which only denote emotional engagement.

The second goal of this work is to understand how the patient and therapist's linguistic styles might relate with moments of alliance rupture. In a study by Mariani et al. (2013) researchers found the therapist and patient differed in their linguistic styles. The patient tended to symbolize more than the therapist, and the therapist tended to reflect and reorganize the speech more than the patient. In another study by Christian, Barzilai, Nyman and Negri (2019), results showed that a strong therapeutic alliance was associated with higher emotional engagement for both patients and therapists, while distancing and defensiveness characterized a weaker alliance. Based on these results we assume there should be linguistic difference between interactions that include ruptures and those that do not. Our goal with this study is to explore whether there are measurable linguistic differences even in the first intake session. We know, generally, that intake sessions are focused on symptom-description, and as mentioned earlier, are likely to contain moderately high symbolizing, so a finding of specific linguistic patterns contributing to the therapeutic alliance even in the first session would be particularly significant. Considering the previous results, moments of rupture are likely to make it difficult for a therapist to maintain a position of reflection, and propel them into a focus on the "here and now." It is also expected that during ruptures the patient will present a decreased connection with their own internal world and a more defensive style of speech.
More specifically, as found by Christian et al. (2019), who investigated the relationship between alliance and referential process within psychotherapy sessions, we assumed that during the intake sessions: i) there would be a positive relation between the capacity to be in contact with emotional experience, (i.e., being immersed in the story telling), reflecting on it, and the quality of therapeutic alliance; ii) conversation segments with high alliance are expected to show a linguistic style denoting higher emotional engagement, whereas weaker alliance segments are expected to be marked by distancing and defensiveness; and finally, iii) the alliance by the end of the intake session, is expected to be predicted by linguistic dimensions denoting a good referential process obtained at the beginning and central part of that same session.

\section{Materials and Methods}

\section{Participants}

Forty patients were selected from a set of 186 videotaped intake sessions conducted in an outpatient psychotherapy center in an Italian hospital. These 15 men and 25 women ranged in age from 18 to $73(M=40.27$, $S D=14.52$ ). Thirty-two participants met criteria for a single diagnosis according to the Diagnostic and Statistical Manual of Mental Disorders, Fifth Edition (DSM-5; American Psychiatric Association, 2013) as follows: Four with panic disorder; three with agoraphobia; one with specific phobia; six with obsessive-compulsive disorder; two with obsessive-compulsive personality disorder; five with bulimia nervosa; two with anorexia nervosa; one with binge eating disorder; and eight with major depressive disorder. Eight participants did not meet criteria for any diagnosis. Seven of those eight individuals presented for treatment to address relationship problems with relatives, and one patient wished to address emotional difficulties relating to voluntary termination of a pregnancy. The selfperceived symptom severity as measured through the Symptomatic Checklist 90-Revised ranged from medium to high for the 32 clinical subjects (Global Severity Index: $M=1.63, S D=0.68$, range $=0.21-3.33$ ) and medium for the eight non-clinical subjects (Global Severity Index: $M=0.60, S D=0.23$, range $=0.26-0.94)$.

The first author conducted all intake sessions, using a semi-structured approach aimed at determining a diagnosis and treatment plan. The sessions lasted from 45 to 80 minutes $(M=54.6, S D=22.1)$ and typically explored, often in sequence, the following topics: i) discussion of the parameters of treatment; referral; reviewing symptoms and other complaints; collecting history of previous psychotherapeutic, physical or pharmacological treatments; ii) opinions of the patient's significant others about symptoms and problems; general discussion of patient's self and significant relationships; iii) motivations for treatment; the patient's expectations about therapy; options for 
treatment; and a first psychological reframing and feedback on the symptoms and problems. All participants signed an informed consent to be videotaped for research purposes.

\section{Procedures}

Three scorers, different from the therapist who conducted the intake sessions, selected the sessions from the available videotapes, assuring a balanced distribution among the four main diagnostic areas considered (phobic, obsessive, eating, and depressive) and the nonclinical group. For the selection process, all of the intake sessions with patients presenting one of these four psychopathologies were extracted. Drawing from the clinical report and the videotape, the scorers then excluded those with psychological comorbidities or non-prototypical symptoms. The first cases that met these inclusion/exclusion criteria were selected until five balanced groups of patients were formed as previously described. The videotaped sessions were transcribed and segmented into five-minute segments for the Segmented Working Alliance Inventory-Observer (S-WAI-O) scoring.

Using other videotaped clinical interactions, scorers were trained on the use of the S-WAI-O until they reached an acceptable group reliability - an interclass correlation (ICC) of .60 or higher (two-way mixed method, with absolute agreement on single measures) calculated on the group scores on each five-minute segment.

Once the group was found to have reached reliability, each scorer coded one third of the sessions independently, based on both, the transcripts and videotapes. The interrater reliability was again checked on 12 sessions that were double-coded; the ICC calculated on the five-minutes segments was .73 .

Each scored segment was classified as either a rupture segment or a non-rupture segment, depending on whether the score for that segment was a 4 or below on the S-WAI$\mathrm{O}$ - the midpoint indicating the neutral valence of the alliance. From the 40 sessions, 33 rupture segments and 288 non-rupture segments were identified. Ten sessions had at least one segment score below 4, and in the other 30 sessions all S-WAI-O scores were above the midpoint.

Finally, the sessions were prepared for the computerized scoring using the referential process measures (Bucci \& Maskit, 2006; Mariani et al., 2013; Negri et al., 2018) described below.

\section{Measures}

\section{Symptom Checklist-90-Revised}

The Symptom Checklist-90-Revised (Derogatis, 1994) is a 90-item self-report symptom inventory that assesses psychological distress in terms of nine primary symptom dimensions (somatization, obsessive-compulsive, interpersonal sensitivity, depression, anxiety, hostility, phobic anxiety, paranoid ideation, and psychoticism) and three summary scores termed global scores (Global Severity Index, the Positive Symptom Distress Index, and the Positive Symptom Total). This measure was administered to participants at the end of the intake interview.

\section{The Segmented Working Alliance Inventory Observer form}

S-WAI-O (Berk, Safran, Muran, \& Eubanks-Carter, 2010) is an observer-based measure of working alliance consisting of 12 items, divided in two scales: agreement on task and agreement on bond. Each of the 12 items is rated on a 7-point scale, with 1 denoting very strong evidence against, 4 no evidence or equal evidence, and 7 very strong evidence for. To rate the S-WAI-O evaluators must watch the videotape of the session; all 12 items are scored at 5-minute intervals. For each 5-minute segment the SWAI-O score is calculated as the average of the 12 scores. Some studies (Berk, 2013; Berk et al., 2010) have found that S-WAI-O correlates moderately with patient self-report alliance inventories, but not with therapist reports.

\section{Computerized linguistic measures of referential process}

The Discourse Attribute Analysis Program (DAAP; Maskit, 2011; Maskit, Bucci, \& Murphy, 2012) is a computer software program that compares any type of text with some lists of words or dictionaries in order to either obtain the proportion in which those words are present in the text or to ascertain the average of the weights they assume relative to a certain construct. DAAP reads texts, compares them word by word to one or more dictionaries, and calculates a weighted average of the dictionary scores for each speaker and each turn of speech, for each text, and for each session. This provides researchers with averages for a micro and macro analysis of sessions. The software also produces a type of derived measure examining the covariations between dictionaries.

Over the last 20 years a number of dictionaries operationalizing the phases of referential process were created and developed in English and in other languages. In our study, we used the following dictionaries and derived measures built and validated for the Italian language.

\section{The weighted dictionaries and derived measures}

\section{Italian Weighted Referential Activity Dictionary}

The Italian Weighted Referential Activity Dictionary (IWRAD) is a computerized measure of RA (Mariani et al., 2013) for the Italian language. It contains a list of 9,596 frequently used Italian words, each assigned a weight between 0 and 1 , with .5 as the neutral value. A high score represents a high level of referential activity, which corresponds to a high level of concreteness, specificity, clarity, and imagery in the speech sample. Part of the value of the IWRAD derives from its power to assess linguistic style (rather than only focusing on content) and to represent the unintended aspects of emotional involvement. Through IWRAD, it is possible to detect and model 
the narrative/symbolizing phase of the referential process.

What follows are two examples of high and low RA excerpts (Bucci, Kabasakalian, \& the Referential Activity Research Group, 2004):

I can't stand fruit with bad spots in it. It gives me the creeps. So I picked up that pineapple and it looked so nice, and then my finger went right through inside it, into this brown, slimy, mushy stuff, and my stomach just turned over. (p. 27).

In this example with high RA, the speaker is describing an experience in such a way that it is easily conjured in imagination - the visual image of the fruit, the texture of the inside, even the physical repulsion felt by the speaker. There is a feeling of a solid, vivid connection to the memory.

I love people and I like to be with people. And right now I feel very bad because I can't be with them and do the things I would like to do. But I'm looking forward to a happier and healthier future and - I don't know what else to say. What else can I talk about? Well - I've had a very eventful life, I think. I've worked practically all my life and I love people. (p. 31).

In this example with low RA, there are many vague references and a feeling that the speaker is not connected to his/her feelings.

\section{Italian Weighted Reflection and Reorganization List}

Reorganization and reflection function refers to the degree to which the speaker is trying to recognize and understand the emotional significance of an event or set of events in their own or someone else's life, or in a dream or fantasy. It is not about abstract reflection but rather a person's reasoning related to an experience that has been vividly experienced. The Italian Weighted Reflection and Reorganization List (IWRRL) is a list of Italian weighted (ranging from 0 and 1, with .5 as a neutral value) words referring to the reorganization and reflection function; the list consists of 1,633 words with coverage of $89 \%$ of the Italian language as it normally spoken (Negri et al., 2018). High scores on this measure represent high reflection/reorganizing as understood through MCT. Through IWRRL, it is possible to detect and model the reorganizing phase of the referential process.

Examples of excerpts with high and low reorganization/reflection function follow (Bucci, Zhou, \& the Referential Activity Research Group, 2018):

I got a feeling it doesn't remind me of anything. I got the feeling in toilet training I must have, something must have, my mother must have been sitting there with her hand out waiting for the goddamn thing, you know, and maybe I, ah, that would be a funny one it's as though that it was a battle between me and my mother who'd get hold of my shit. She wanted it and I wanted it. She wanted it, by getting it she had possession of me, or something. I don't know. Ah let's see, if I get hold of it and hid it, then she couldn't have it, could she? And ah, if she couldn't have it, then I was safe. Ah, I don't know. (p. 2).

In this example the speaker is trying to actively search subjective meanings related to a memory in which she/he is emotionally engaged.

I had tried to clean the bedroom floor for her. I went into the kitchen. It was after my sister was born. My sister was already on the scene. I went into the kitchen and took out about three cans of Ajax or whatever was the type of detergent used at the time and proceeded to open up the cans and dump all the soap powder on the floor and smoosh it around and going off to tell my mother what a wonderful thing I had done. (p. 3).

Here the speaker is just describing a memory without any active effort of reflection and reorganization.

\section{Mean High - Italian Weighted Referential Activity Dictionary}

The Mean High - Italian Weighted Referential Activity Dictionary (MH-IWRAD) is calculated on IWRAD scores and is a measure of high intensity of emotional engagement (Mariani et al., 2013) emerging from the speech. It indicates how high the IWRAD is when it is above the mean: It is obtained by looking only at the words with IWRAD scores lying above the neutral value and then computing, for only those words, the average amount of the IWRAD scores. This is perhaps best understood as a measure of upward oscillations in RA scores.

\section{Mean High - Italian Weighted Reflection and Reorganization List}

The Mean High - Italian Weighted Reflection and Reorganization List (MH-IWRRL) is calculated on IWRRL scores and is a measure of the high intensity of the reflection and reorganization function as emerges from a person's speech (Negri et al., 2018). It indicates how high the IWRRL is when it is above the mean: It is obtained by looking only at the words with IWRRL scores lying above the neutral value and then computing, for these words only, the average amount of the IWRRL scores. It is best understood as a measure of the upward oscillations in reflection and reorganizing scores.

\section{The unweighted dictionaries}

Some example words from each dictionary are provided in Table 1.

\section{Italian Reflection Dictionary}

The Italian Reflection Dictionary (IRefD) consists of Italian words referring to cognitive or logical functions, and to communication processes that imply the use of cognitive functions (Mariani et al., 2013). It is a measure of abstract reflection and distancing from emotional experience and corresponds to the proportion of IRefD words present in the speech. 


\section{Italian DisFluency Dictionary}

The Italian DisFluency Dictionary (IDFD) is a small set of words as well as repeated words, incomplete words and filled pauses that people tend to use when struggling to communicate (Bonfanti, Campanelli, Ciliberti, Golia, \& Papini, 2008; Maskit et al., 2012). This index corresponds to the proportion of IDFD words present in the speech. High scores typically characterize the arousal phase in which the emotion schemas are activating.

\section{Italian Sensory Somatic Dictionary}

The Italian Sensory Somatic Dictionary (ISensD) is a list of Italian words related to the body and bodily activities, and to sensory processes and/or descriptions of symptoms (Di Trani, Mariani, Renzi, Greenman, \& Solano, 2018). The number of ISensD words in the speech sample is a measure of the arousal of bodily subsymbolic aspects of emotion schemas.

\section{Italian Affect Dictionary}

The Italian Affect Dictionary (IAffD) contains Italian words concerning how people feel and communicate feelings directly. It includes emotion labels; functions associated with affective arousal; functions indicating motivation; words implicitly associated with affect; and evaluations indicating an affective response, either positive or negative. IAffD consists of four sub-dictionaries related to domains of affect: positive affects (IPAffD); negative affects (INAffD); neutral affects without a specific valence (IZAffD); the sum of the other measures (ISAffD). The proportions of words of each dictionary present in the speech form the four indices related to the affects words.

\section{Data analysis}

Since alliance and linguistic style tend to vary repeatedly and not always in a linear way during a psychotherapy session, we decided to divide each session into three parts (initial, central, and final) to evaluate the diachronic progression, piece by piece, of the linguistic measures in connection with the therapeutic alliance. We analyzed this progression using a correlational analysis of the 40 participant scores in each third of the sessions using Pearson correlation coefficients.

For our second analysis, we divided the 40 sessions into two groups, depending on whether a rupture occurred during the session: the non-rupture group was defined by score of 4 or above on the S-WAI-O on all segments; while the rupture group was defined by at least one segment with an alliance score below 4 . We compared the two groups along the language indices and conducted independent sample $t$-tests to analyze differences between the groups based on our referential process measures. We chose the strongest Welch's $t$ statistic since we had an unbalanced research design and heteroskedasticity was present in some of the variables (Delacre, Lakens, \& Leys, 2017).

A final analysis was carried out to verify whether it was possible to predict the alliance in the last part of the session by taking into account the linguistic measures collected from the first and second parts of the session. A linear multiple regression analysis was carried out using the linguistic dimensions measured in the first two thirds of the session as predictors of the S-WAI-O score of the last third. A model explaining the greater variance of the target variable was retained.

Having found no difference in the S-WAI-O scores between participants with a DSM diagnosis $(n=32)$ and nonclinical ones $(n=8)$ we did not differentiate the two groups in the three analyses conducted.

\section{Results}

\section{Linguistic measures and alliance throughout the intake session}

When the patients' speech was considered, some associations between the referential process measures and

Table 1. Examples of words contained in the unweighted Italian dictionaries of the referential process.

IRefD Attenzione (attention), capire (to understand), decisione (decision), dubbio (doubt), meditare (to meditate), ragione (reason), razionalità (rationality), ricordo (memory).

IDFD Quindi (so), cioè (that is), comunque (however), allora (then), insomma (well), niente (nothing), magari (maybe), vabbè (don 't care), boh (don't know), and 'ehmm' and ' $m m$ ' representing filled pauses with slightly different significance.

ISenSD Ammalato (sick), digerire (digest), disorientamento (disorientation), dolore (pain), impotente (impotent), innervosito (unnerved), pesare (weigh), ridere (laugh), sintomi (symptoms), vomitare (throw up).

INAffD Abbandonato (abandoned), depresso (depressed), impaurito (frightened), invidioso (envious), malinconia (gloom), odio (hate), sofferenza (suffering).

IPAffD Abbracci (hugs), affidabile (reliable), baciare (to kiss), felice (happy), innamorato (in love), speranza (hope).

IZAffD Attesa (expectation), bisogno (need), coinvolto (involved), eccitato (excited) intensità (intensity), motivazione (motivation), reagire (react), sensazione (feeling).

IRefD = Italian Reflection Dictionary; IDFD = Italian Disfluency Dictionary; ISenSD = Italian Sensory-Somatic Dictionary; IPAffD, INAffD, IZAffD = Italian Dictionary of respectively Positive, Negative, neutral (Z) Affects. 
the quality of the alliance were found (Figure 1). At the beginning of the intake sessions there was one significant negative correlation between S-WAI-O and ISenSD $(r=-$ $.35, \mathrm{P}<.05)$. The more patients referred to the somatic and physical sensations - for example talking about the somatic aspects of their symptoms - the lower the alliance.

In the middle third of the sessions, linguistic measures showed a different pattern. S-WAI-O was positively correlated with the number of words produced by patient $(r=.46$, $\mathrm{P}<.01)$ and negatively correlated with IWRRL $(r=-.33$, $\mathrm{P}<.05)$, MHIWRAD $(r=-.36, \mathrm{P}<.05)$, MHIWRRL $(r=-.37$, $\mathrm{P}<.05)$. Interactions in which patients displayed lower reorganization and reflection activity as well as lower RA intensity were associated with a stronger alliance.

The final part of the intake sessions was characterized by a positive correlation between S-WAI-O and total number of words $(r=.33, \mathrm{P}<.05)$ and by a negative correlation between S-WAI-O and IZAffD $(r=-.43, \mathrm{P}<.01)$. In other words, a stronger alliance was associated with a patient's longer speech during this phase, with fewer words referring to neutral affects.

In all three parts of the session, there was no association found between the therapists' speech and either the alliance or linguistic measures.

\section{Linguistic style in rupture and non-rupture sessions}

A comparison between rupture $(n=10)$ and non-rupture sessions $(n=30)$ showed significant in differences in the S-WAI-O index (Welch's $t(21.77)=10.67, \mathrm{P}<.001$, $d=4.52$ ) and in a few linguistic measures (Table 2): IDFD of patient (Welch's $t(20.86)=4.09, \mathrm{P}<.001, d=0.14$ ) and therapist (Welch's $t(10.67)=4.87, \mathrm{P}<.001, d=2.57$ ) was higher in the non-rupture sessions, as was the therapist's IRefD (Welch's $t(2.18)=11.55, \mathrm{P}<.05, d=0.09$ ); the patient's IZAffD (Welch's $t(-3.03)=11.67, \mathrm{P}<.05, d=0.12$ ) was higher in the rupture sessions. In sum, rupture sessions, both patient and therapist had speech marked by fewer disfluencies; with more of the patients' words referring to neutral affects and fewer of the therapists' words referring to abstract reflection.

\section{Linguistic predictors of alliance}

The results of our multiple regression analysis indicate that four patients' linguistic dimensions in the second third of sessions explained $54.5 \%$ of the variance in the S-WAI$\mathrm{O}$ score of the final third of the session $\left(R^{2}=.545\right.$, $F(4,35)=10.46, \mathrm{P}<.001)$. S-WAI-O in the last third of the session was predicted by a combination of MHIWRRL $(\beta=-4.24, t=-5.11, \mathrm{P}<.001)$, IWRRL $(\beta=3.97, t=4.77$, $\mathrm{P}<.001)$, MHIWRAD $(\beta=-0.33, t=-2.81, \mathrm{P}<.01)$, and IRefD $(\beta=-0.24, t=-2.04, \mathrm{P}<.05)$ as measured in the second third of the sessions. Specifically, a stronger alliance at the end of the session was predicted by speech in the middle of the session, which was characterized by high reorganization and reflection on the emotional elements and low abstract reflection and at the same time RA and

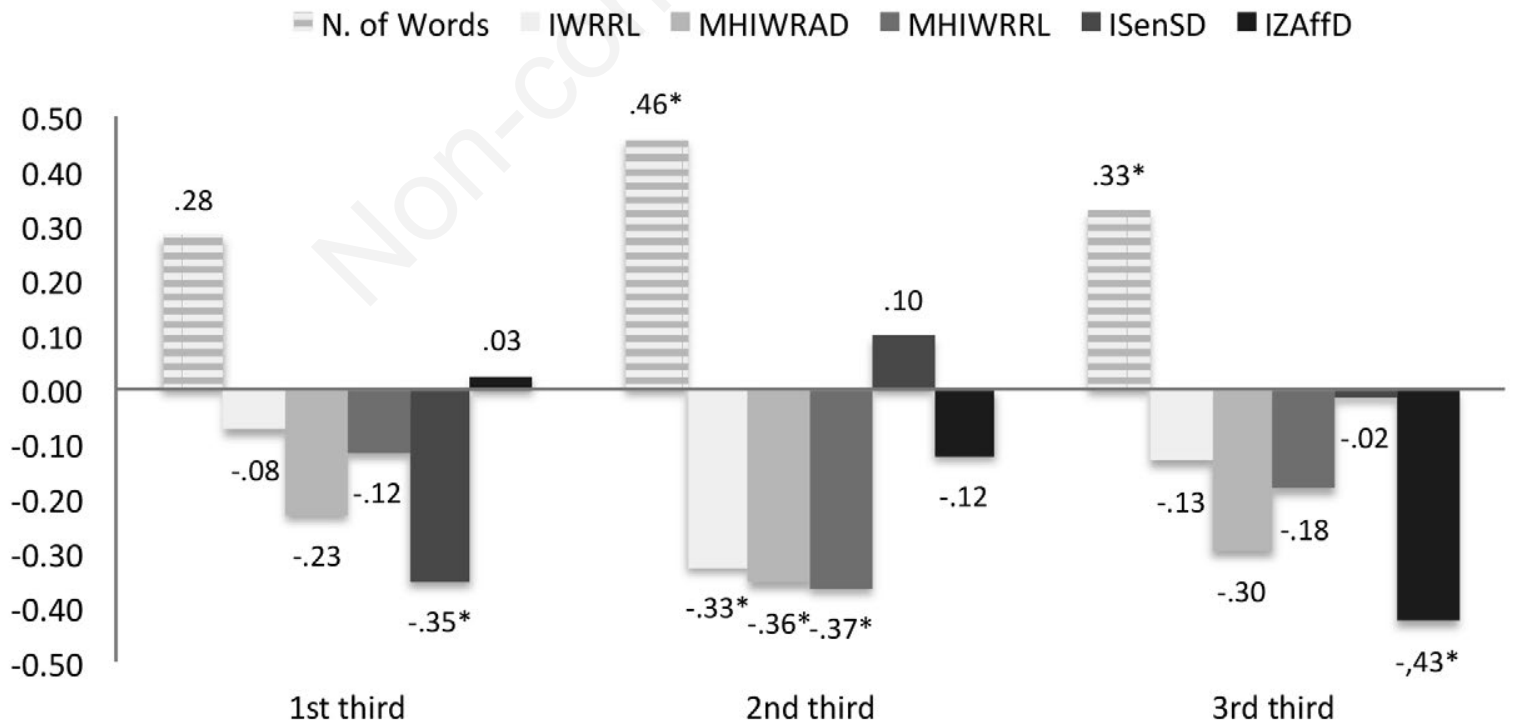

Figure 1. Pearson correlations between Segmented Working Alliance Inventory Observer-based scores and some linguistic measures of patients' speech in three thirds of the sessions from 40 treatments considered. N. of Words = Number of Words; IWRRL = Italian Weighted Reorganization and Reflection List; MHIWRAD = Mean High Italian Weighted Referential Activity Dictionary; MHIWRRL = Mean High Italian Weighted Reorganization and Reflection List; ISenSD = Italian Sensory-Somatic Dictionary; IRefD = Italian Reflection Dictionary; IZAffD = Italian neutral (Z) Affects Dictionary. Next to the bars are reported the coefficients. $* \mathbf{P}<.05$. 
reorganization/reflection showed no high elevations when they were above the mean.

Regarding the therapists' speech, no dimension of their linguistic style in the initial and central parts of the intake sessions predicted the alliance level in the final part.

\section{Discussion}

The correlational analysis in the intake sessions showed distinct patterns of linguistic styles according to three phases of the sessions analyzed.

In the beginning phase, if the patient made fewer references to body, sensory processes (perceptual, visceral and kinesthetic), and bodily activities, the alliance was found to be stronger. It is important to note that the cultural or clinical context, as well as the way the therapist conducts the intake session can certainly lead patients to focus first on their symptoms. However, we found the more this occurred, the more the alliance tended to be weaker. It is possible that this focus on symptoms and somatic experience reflects a defensive action in the new relational situation that is just being established.

In the mid-phase of the intake session, the more the patients speak and take time to express something about their life, the stronger the alliance; but a greater alliance was associated also with a lower reorganization and reflection activity and lower RA intensity. This means that if during the mid-phase of the intake the patients take time to talk about themselves, the alliance improves; but this should involve neither excessive involvement nor an intense work of reflection and reorganization on what has been told.

In the last part of session, a greater alliance was associated to a patients' longer speech containing less words referring to neutral affects. A high expression of neutral affects denotes emotional control and/or defensiveness, since a speaker refers to emotions, but without a clear va- lence. By the end of the intake session, a less defensive linguistic style is connected to higher alliance. By contrast, a patient who is not able to be more specific in describing their affects, remains vague and describes experience in generic terms, ultimately is less involved in the therapeutic relationship.

In sum, the correlation pattern indicated that if during the intake session patients were not too focused on body and symptoms; took time to talk about themselves without too much intense activation; moderated their attempts at reorganization; and finally described their emotions and affects in non-neutral terms, then the therapeutic alliance tended to be stronger.

The comparison between the alliance rupture and nonrupture sessions highlighted some important features of the alliance. During non-rupture sessions, both conversational partners presented more disfluency, patients used less words referring to affects, and therapist used more words referring to communication and thought function. In particular, the disfluent style is a very interesting aspect of referential process, which is strictly related to arousal phase. The arousal phase is when a problematic emotion schema is activated. In this situation, the patient appears to be struggling with painful feelings and ideas associated with the schema. The subsymbolic processing system, involving bodily and sensory experience, is dominant; this constitutes the affective core of the schema. In other words, a good alliance in the first session allows the patient to begin making contact with their internal world. This finding from the referential process points to the first connection the patient makes with a problematic emotional schema. Of course, in the first session the patient is unlikely to be able to go deeper into a symbolizing or reflecting phase. But, in all, the more the patient uses affect words (regardless of whether they are neutral, positive, or negative) the lower the alliance. In fact, just labeling emotions rather than engaging in vivid description of them is a sign of distancing and defense: In this case the use of

Table 2. Comparison between rupture and non-rupture intake sessions.

\begin{tabular}{|c|c|c|c|c|c|c|c|c|c|c|c|c|c|}
\hline Spk & Sessions & & IWRAD & IWRRL & MHIWRAD & MHIWRRL & IRefD & IDFD & ISenSD & IPAffD & INAffD & IZAffD & ISAffD \\
\hline \multirow[t]{4}{*}{$\mathrm{Pt}$} & Rupture $(n=10)$ & $M$ & .502 & .539 & .012 & .040 & .022 & .049 & .051 & .013 & .016 & .008 & .037 \\
\hline & & $D S$ & .006 & .004 & .003 & .004 & .011 & .019 & .014 & .008 & .010 & .006 & .008 \\
\hline & Non-rupture $(n=30)$ & $M$ & .501 & .540 & .010 & .041 & .020 & .079 & .047 & .010 & .014 & .005 & .029 \\
\hline & & $D S$ & .003 & .002 & .002 & .002 & .006 & .024 & .011 & .003 & .004 & .002 & .005 \\
\hline \multirow[t]{4}{*}{$\mathrm{Tp}$} & Rupture $(n=10)$ & $M$ & .504 & .545 & .012 & .046 & .021 & .045 & .047 & .008 & .017 & .004 & .029 \\
\hline & & $D S$ & .002 & .006 & .003 & .006 & .007 & .014 & .014 & .004 & .014 & .004 & .017 \\
\hline & Non-rupture $(n=30)$ & $M$ & .502 & .548 & .013 & .049 & .029 & .071 & .051 & .010 & .017 & .006 & .033 \\
\hline & & $D S$ & .003 & .004 & .002 & .003 & .011 & .014 & .013 & .003 & .014 & .002 & .006 \\
\hline
\end{tabular}

Spk = Speaker; Pt = Patient; Tp = Therapist; IWRAD = Italian Weighted Referential Activity Dictionary; IWRRL = Italian Weighted Reflection and Reorganization List; MHIWRAD = Mean High Italian Weighted Referential Activity Dictionary; MHIWRRL = Mean High Italian Weighted Reflection and Reorganization List; IRefD = Italian Reflection Dictionary; IDFD = Italian Disfluency Dictionary; ISenSD = Italian Sensory-Somatic Dictionary; IPAffD, INAffD, IZAffD = Italian Dictionary of respectively Positive, Negative, neutral (Z) Affects; ISAffD = Italian Dictionary Sum of Affects (IPAffD, INAffD, IZAffD). The significantly higher values in the comparisons are in bold. 
general and abstract words to describe the emotional sensations is a way to ineffectively fill the gap perceived between subsymbolic and symbolic experience (Bucci, Maskit, \& Murphy, 2016; Izard, Stark, Trentacosta, \& Schultz, 2008; Lieberman et al., 2007; Tabibnia, Lieberman, \& Craske, 2008).

In conclusion, we can say that a patient's use of either a disfluent style or affect words reflects two opposite ways of handling the activation of emotional patterns in the first session: Disfluency indicates an emergence of emotional contents and an attempt to translate them into words, while naming the feelings in an abstract manner indicates a form of intellectualization.

Interestingly, higher disfluency in the therapist was also evidence of stronger alliance. This could indicate a very interesting sub-symbolic relationship between patient and therapist. So, an emotion schema is sub-symbolically activated in the relationship and something happens under the surface of their formal speech. The greater amount of reflection words used contemporarily by the therapist could be interpreted as an attempt at a first abstract understanding of the patient's experience - one that can only be emotionally-deepened in subsequent phases of treatment. These results are consistent with findings from Rocco, Gennaro, Salvatore, Stoycheva, and Bucci (2017), who showed that a good-outcome psychotherapy session is characterized by a process marked by attunement, which over time promotes the integration of emotional and cognitive domains of speech.

The regression analysis showed a predictive function between the linguistic measures and the alliance within the intake session. As expected, the emotional reorganization and non-abstract reflection are those elements that most favor the alliance in the first session - more than the referential activity itself. As we mentioned, RA tends to be high during an intake session because the patients are emotionally activated from the fact that they are attending a first meeting and from the act of describing their symptoms and state of suffering. However, if by the middle phase of the session, patients reflected and reorganized experience that was vivid but not too intense, then the strength of the alliance increased by the end of that session.

This result seems particularly important to us because it offers a different perspective on the factors that contribute to the alliance. As pointed out by other authors (Johnsson \& Stenlund, 2010; Safran \& Kraus, 2014; Safran \& Muran, 2000), the therapeutic alliance is not the result of a rational and conscious effort of agreement between patient and therapist, but rather a process of joint emotional elaboration of the issues emerging in the therapeutic exchange. Such elaboration could at first disorient the patient who does not find his collusive expectations confirmed; however, precisely because the dyad jointly produces new, significant, and unexpected meanings, they strengthen the alliance.

It is also important to underline that this study has re- vealed that it is possible, as early as the intake session, to differentiate and predict high and low alliance by analyzing the linguistic style of patient and therapist. The intervention of the therapist in the first session is certainly less pronounced than in the subsequent phases but even at this early phase their role is already fully active. This finding is interesting from various points of view: Clinically, because it indicates the importance of the early elaboration process with the patient in order to positively activate the therapeutic relationship; conceptually, inasmuch to differentiate markedly an initial phase of diagnosis and a subsequent phase of intervention it may not be justified and lead to an arbitrary distance from the actual process already ongoing from the very early first meeting.

One important limitation of the study is, of course, that only one therapist conducted all intake sessions. This fact reduces the variability linked to the therapist and therefore the generalizability of the results. For this reason we invite researchers to replicate the study with different patients and different therapists, and also analyze sessions subsequent to the intake. However, we believe that three elements counterbalanced and limited the effect of the lower variability related to the therapist: i) the variability related to patients was intentionally increased as much as possible by recruiting five balanced groups different in terms of their diagnosis; so patients with supposedly different relational style responded differently even if they faced with the same therapist and with a similar interview; ii) we analyzed the first intake session that is the very first meeting with the therapeutic system; the two conversational partners do not yet know each other and they both do not know if they will embark in a treatment or not. We think that in this very first phase the therapist remains in the background while the patient with her/his request and suffering takes the prominent space and role. Only afterwards does the relationship take on a more balanced nature as the therapist assumes a greater influence on the progress of the communicative and interactive processes; iii) we also analyzed the interventions of the therapist and no significant results were found. The one difference is that therapist used more abstract reflection words in non-rupture sessions. We can therefore infer that the interventions of the therapist have been relatively homogeneous both within the session and with the different patients and that the variations detected are to be attributed more to the characteristics of the patients. Furthermore, all patients are faced with a similar situation (same therapist and same semi-structured interview) so the significant variations detected in correlation, regression and difference analyses do not seem to be due to the therapist or to the difference in the approach to conducting the intake sessions but to the variability of patients showing high or low scores in alliance and linguistic measures.

Results from this study certainly need to be confirmed by other studies, employing larger samples of intake sessions, and conducted by different therapists, from differ- 
ent theoretical perspectives; furthermore, it would be necessary to verify if the linguistic style of the intake session has any value in predicting the subsequent psychotherapy process and the ultimate outcome of a treatment. In spite of these limitations, however, this study is one in a growing line of research that analyzes how subjects speak rather than just the content of what they say to expand our understanding of the alliance and other factors that account for change in treatment.

\section{Conclusions}

The results suggest a new understanding of the underpinning elements of alliance processes, at least for how they occur in the intake session. The linguistic style of the therapeutic dyad reveals that the activation of emotion schemas in the session and the ability of the therapeutic dyad to symbolize, reorganize, and reflect on this shared emotional experience fosters a confidence in the ability to work together that can be measured as therapeutic alliance; a good therapeutic alliance in turn should impact the next symbolizing and reflecting phase.

The implication for clinical practice is that already in the intake session therapists should give room to patients to immerge in the emotional experience and reflect on it; the agreement on the task and bond is likely an effect of this work of immersion and elaboration.

\section{References}

American Psychiatric Association (2013). Diagnostic and statistical manual of mental disorders. 5th edition (DSM-5). Washington, D.C., US: APA Association.

Aron, L. (1996). A meeting of minds: Mutuality in psychoanalysis. Hillsdale, NJ, US: Analytic Press.

Berk, E. A. (2013). Detecting alliance ruptures and rupture repair with the segmented working alliance inventory-observer form (S-WAI-O). Dissertation Abstracts International: Section B. The Sciences and Engineering, 74(10), 3566428.

Berk, E. A., Safran, J. D., Muran, J. C., \& Eubanks-Carter, C. E. (2010). The segmented working alliance inventory-observer-based (S-WAI-O) measure: Manual. New York, NY, US: Beth Israel Medical Center.

Bonfanti, A. A., Campanelli, L., Ciliberti, A., Golia, G., \& Papini, S. P. (2008). Speech Disfluency in spoken language: The Italian computerized dictionary (I-Df) and its application on a single case. Paper presented at $39^{\text {th }}$ International Meeting of the Society for Psychotherapy Research, Barcelona, Spain.

Bucci, W. (1984). Linking words and things: Basic processes and individual variation. Cognition, 17, 137-153. doi: 00100277(84)90016-7

Bucci, W. (1997). Psychoanalysis and cognitive science: A multiple code theory. New York, NY, US: Guilford Press.

Bucci, W. (2000). The need for a psychoanalytic psychology in the cognitive science field. Psychoanalytic Psychology, 17, 203-224. doi: 10.1037/0736-9735.17.2.203

Bucci, W. (2002a). Multiple code theory and the referential process. Applications to process research. In P. Fonagy
(Ed.), An open door review of outcome studies in psychoanalysis (2nd ed., pp. 192-195). London, UK: International Psychoanalytical Association.

Bucci, W. (2002b). Referential activity (RA): Scales and computer procedures. In P. Fonagy et al. (Eds.), An open door review of outcome studies in psychoanalysis (2nd ed., pp. 286-288). London, UK: International Psychoanalytical Association.

Bucci, W. (2005). Basic concepts and methods of psychoanalytic process research. In E. Person, A. Cooper, \& G. Gabbard (Eds.), Textbook of psychoanalysis (pp. 339-355). Washington, D.C., US: American Psychiatric.

Bucci, W., \& Freedman, N. (1978). Language and hand: The dimension of referential competence. Personality, 46 , 594-622. doi: 10.1111/j.1467-6494.1978.tb00188.x

Bucci, W., Kabasakalian, R., \& the Referential Activity Research Group (2004). Instructions for scoring referential activity $(R A)$ in transcripts of spoken narrative texts. Available from http://www.thereferentialprocess.org

Bucci, W., \& Maskit, B. (2006). A weighted dictionary for Referential Activity. In J. G. Shanahan, Y. Qu \& J. Wiebe (Eds.), Computing attitude and affect in text (pp. 49-60). Dordrecht, Netherlands: Springer.

Bucci, W., \& Maskit, B. (2007). Beneath the surface of the therapeutic interaction: The psychoanalytic method in modern dress. Journal of the American Psychoanalytic Association, 55, 1355-1397. doi: 10.1177/000306510705500412

Bucci, W., Maskit, B., \& Murphy, S. (2016). Connecting emotions and words: The referential process. Phenomenology and the Cognitive Sciences, 15, 359-383. doi: 10.1007/s11097-0159417-z

Bucci W., Zhou, Y., \& the Referential Activity Research Group (2018). Instructions for scoring the Reflection/Reorganization (RR) Scale. Unpublished manuscript, Parcella Research Center, New York Psychoanalytic Society and Institute, New York, NY, US.

Castonguay, L. G., Constantino, M. J., \& Holtforth, M. G. (2006). The working alliance: Where are we and where should we go? Psychotherapy: Theory, Research, Practice, Training, 43(3), 271. doi: 10.1037/0033-3204.43.3.271

Christian, C., Barzilai, E., Nyman, J., \& Negri, A. (2019). Assessing key linguistic dimensions of ruptures in the therapeutic alliance. Journal of Psycholinguistic Research [in press].

Colli, A., Gentile, D., Condino, V., \& Lingiardi, V. (2017). Assessing alliance ruptures and resolutions: Reliability and validity of the Collaborative Interactions Scale-Revised Version. Psychotherapy Research, 1-14. doi: 10.1080/ 10503307.2017.1414331

Delacre, M., Lakens, D., \& Leys, C. (2017). Why psychologists should by default use Welch's t-test instead of Student's ttest. International Review of Social Psychology, 30, 92-101. doi: 10.5334 irsp.82

Derogatis, L. R. (1994). SCL-90-R administration, scoring, and procedures manual (3 ${ }^{\text {rd }}$ Ed.). Minneapolis, MN: NCS Pearson Inc.

Di Trani, M., Mariani, R., Renzi, A., Greenman, P. S., \& Solano, L. (2018). Alexithymia according to Bucci's multiple code theory: A preliminary investigation with healthy and hypertensive individuals. Psychology and Psychotherapy: Theory, Research and Practice, 91, 232-247. doi: 10.1111 papt. 12158

Emde, R. N. (1983). The prerepresentational self and its affec- 
tive core. The Psychoanalytic Study of the Child, 38, 165-192. doi: 10.1080/00797308.1983.11823388

Eubanks, C. F., Muran, J. C. \& Safran, J. D. (2018). Alliance rupture repair: A meta-analysis. Psychotherapy, 55, 508519. doi: 10.1037/pst0000185

Flückiger, C., Del Re, A. C., Wampold, B. E. \& Horvath, A. O. (2018). The alliance in adult psychotherapy: A meta-analytic synthesis. Psychotherapy, 55, 316-340. doi: 10.1037 /pst0000172

Horvath, A. O. (2005). The therapeutic relationship: Research and theory. Psychotherapy Research, 15, 3-7. doi: 10.1080/10503300512331339143

Horvath, A. O., Del Re, A. C., Flückiger, C., \& Symonds, D. (2011). Alliance in individual psychotherapy. Psychotherapy, 48, 9-16. doi:10.1037/a0022186

Izard, C., Stark, K., Trentacosta, C., \& Schultz, D. (2008). Beyond emotion regulation: Emotion utilization and adaptive functioning. Child Development Perspectives, 2, 156-163. doi: 10.1111/j.1750-8606.2008.00058.x

Johnsson, R., \& Stenlund, G. (2010). The affective dimension of alliance in transactional analysis psychotherapy. International Journal of Transactional Analysis Research, 1, 45-59. doi: $10.29044 / \mathrm{v} 1 \mathrm{ilp} 45$

Kingsley, G. (2010). The clinical validation of measures of the referential process. Dissertation Abstracts International: Section B. Sciences and Engineering, 70(9-B), 3377938.

Kris, E. (1956). On some vicissitudes of insight in psychoanalysis. The International Journal of Psychoanalysis, 37, 445-455.

Lambert, M. J. (Ed.). (2013). Bergin and Garfield's handbook of psychotherapy and behavior change. New York, NY, US: Wiley \& Sons.

Lambert, M. J., \& Barley, D. E. (2001). Research summary on the therapeutic relationship and psychotherapy outcome. Psychotherapy: Theory, Research, Practice, Training, 38, 357. doi: 10.1037/0033-3204.38.4.357

Lieberman, M. D., Eisenberger, N. I., Crockett, M. J., Tom, S. M., Pfeifer, J. H., \& Way, B. M. (2007). Putting feelings into words. Psychological Science, 18(5), 421-428. doi: 10.1111/j.1467-9280.2007.01916.x

Mariani, R., Maskit, B., Bucci, W., \& De Coro, A. (2013). Linguistic measures of the referential process in three short term psychodynamic treatments: The Italian version. Psychotherapy Research, 23, 430-447. doi: 10.1080/10503307. 2013.794399

Maskit, B. (2011). DAAP Math I: Word Count Base. Available from: https://docs.google.com/file/d/0B3goZAni6zBkMGI-
wODExOGUtZDgzNi00ODNjLTk0NjEtZj11Zjg1ZjJhMjQ1/edit?pli_1

Maskit, B., Bucci, W., \& Murphy, S. (2012). Computerized Language Measures. Available from: https://sites.google.com/ site/referentialprocess/dictionary-measuresand-computer-programs

Mitchell, S. A. (1998). Relational concepts in psychoanalysis. Cambridge, MA, US: Harvard University Press.

Negri, A., Esposito, G., Mariani, R., Savarese, M., Belotti, L., Squitieri, \& B., Bucci, W. (2018). The Italian weighted reflection and reorganization list (I-WRRL): A new linguistic measure detecting the third phase of the referential process. Research in Psychotherapy: Psychopathology, Process and Outcome, 21(s1), 5-6.

Okie, J. E. (1991). Action, somatization and language in borderline inpatients. Dissertation Abstracts International: Section B. Sciences and Engineering, 53(3-A), 9211084.

Rocco, D., Gennaro, A., Salvatore, S., Stoycheva, V., \& Bucci, W. (2017). Clinical mutual attunement and the development of therapeutic process: A preliminary study. Journal of Constructivist Psychology, 30, 371-387. doi: 10.1080/10720537.2 016.1227950

Safran, J. D. (2012). Psychoanalysis and psychoanalytic therapies. Washington, D.C., US: American Psychological Association.

Safran, J. D., \& Kraus, J. (2014). Alliance ruptures, impasses, and enactments. A relational perspective. Psychotherapy, 51, 381-387. doi: 10.1037/a0036815

Safran, J. D., \& Muran, J. C. (2000). Negotiating the therapeutic alliance: A relational treatment guide. New York, NY, US: Guilford.

Safran, J. D., Muran, J. C., \& Eubanks-Carter, C. (2011). Repairing alliance ruptures. Psychotherapy, 48, 80-87. doi: 10.1037/a0022140

Sexton, H., Littauer, H., Sexton, A., \& Tømmerås, E. (2005). Building an alliance: Early therapy process and the clienttherapist connection. Psychotherapy Research, 15, 103-116. doi: 10.1080/10503300512331327083

Tabibnia, G., Lieberman, M. D., \& Craske, M. G. (2008). The lasting effect of words on feelings: Words may facilitate exposure effects to threatening images. Emotion, 8, 307-317. doi: 10.1037/1528-3542.8.3.307

Zilcha-Mano, S., \& Errázuriz, P. (2017). Early development of mechanisms of change as a predictor of subsequent change and treatment outcome: The case of working alliance. Journal of Consulting and Clinical Psychology, 85(5), 508-520. doi: 10.1037/ccp0000192.supp 